\title{
AVERSÃO AO RISCO, EFEITO ESCALA E EFEITO INCENTIVO: UM EXPERIMENTO LABORATORIAL COM ALUNOS DA UFC
}

\author{
RISK AVERSION, LEVEL AND INCENTIVE EFFECTS: A \\ LABORATORIAL EXPERIMENT WITH UFC STUDENTS
}

\section{AVERSIÓN AL RIESGO, NIVEL Y INCENTIVO EFECTO: UN EXPERIMENTO LABORATORIAL CON ALUMNOS DE UFC}

\author{
Ricardo Brito Soares \\ PhD em Economia pela University of New \\ Hampshire, EUA \\ Professor associado da Universidade Federal do \\ Ceará, Brasil \\ ricardosoares@caen.ufc.br

\section{Patrícia Simões} \\ Doutora em Economia Universidade Federal do \\ Ceará, Brasil \\ pati_simoes@hotmail.com \\ Paulo de Melo Jorge Neto \\ $\mathrm{PhD}$ em Economia pela University of Illinois at \\ Urbana Champaign, EUA \\ Professor associado da Universidade Federal do \\ Ceará, Brasil \\ pjneto@caen.ufc.br
}

Contextus

\section{RESUMO}

Este trabalho faz um estudo empírico sobre tomada de decisão sob incerteza junto a alunos da Universidade Federal do Ceará. Para mensurar as preferências individuais de risco, utiliza-se a técnica de elicitação denominada Lista De Preços Múltiplos (Multiple Price Lists). Tal metodologia, popularizada por Holt e Laury (2002), faz uso de uma lista de loterias pareadas, estruturada de maneira que o número de escolhas seguras do indivíduo possa ser utilizado para estimar o seu grau de aversão ao risco. Como resultado, o nível de aversão ao risco encontrado é maior que o previsto pela teoria, e as hipóteses de efeito da escala de valores e efeito do incentivo monetário são validadas pelo experimento. Desse modo, este trabalho constata uma tendência de subestimação na disposição dos indivíduos em assumir risco em experimentos hipotéticos de laboratório, com possibilidade de prejuízos econômicos.

Palavras-chave: Aversão ao risco; Loteria; Experimento laboratorial; Efeito escala; Efeito incentivo.

\begin{abstract}
This paper makes an empirical study about decision making under uncertainty among students of the Federal University of Ceará. In order to measure individual risk preferences, an elicitation technique called the Multiple Price Lists is used. Such a methodology was popularized by Holt and Laury (2002) and makes use of a list of paired lotteries, structured in such a way that the number of safe choices of the individual can be used to estimate his or her degree of risk aversion. As a result, the level of risk aversion found is higher than predicted by the theory and the hypothesis of the value scale effect as well as that of the monetary incentive effect are validated by the experiment. Thus, this work shows that there is a tendency to underestimate the willingness of individuals to take risks in hypothetical laboratory experiments, with the possibility of economic losses.
\end{abstract}

Key-words: Risk aversion; Lottery; Laboratory experiment; Scale effect; Incentive effect. 


\section{RESUMEN}

Este trabajo hace un estudio empírico sobre toma de decisión bajo incertidumbre junto a alumnos de la Universidad Federal de Ceará. Para medir las preferencias individuales de riesgo, se utiliza la técnica de selección denominada Lista de Precios Múltiples (Multiple Price Listas). Esta metodología fue popularizada por Holt y Laury (2002) y hace uso de una lista de loterías pareadas, estructurada de manera que el número de opciones seguras del individuo pueda ser utilizado para estimar su grado de aversión al riesgo. Como resultado, el nivel de aversión al riesgo encontrado es mayor que el previsto por la teoría, y las hipótesis de efecto de la escala de valores y efecto del incentivo monetario son validadas por el experimento. De ese modo, este trabajo constata una tendencia a la subestimación en la disposición de los individuos a asumir riesgo en experimentos hipotéticos de laboratorio, con posibilidad de pérdidas económicas.

Palabras clave: Aversión al riesgo; Lotería; Experimento de laboratorio; Efecto de escala; Efecto de incentivo.

\section{INTRODUÇÃO}

A atitude do tomador de decisões diante de um risco exerce papel fundamental em vários cenários econômicos, especialmente nos mercados financeiro e de trabalho. Ao, por exemplo, ofertar um seguro, o intermediário financeiro precisa conhecer o grau de aversão ao risco de seu cliente para calcular o prêmio de modo adequado. Torna-se necessário então obter empiricamente a preferência dos indivíduos de assumirem mais ou menos risco.

Nesse contexto, este trabalho tem como objetivo principal fazer um estudo empírico sobre tomada de decisão sob incerteza. Como objetivos específicos, pretende-se estimar um indicador de grau de aversão ao risco para indivíduos e verificar se ele pode ser alterado circunstancialmente por variações nas ofertas monetárias escalonadas hipoteticamente (efeito escala) ou em termos reais (efeito incentivo). Pretende-se, portanto, contribuir com um estudo de caso empírico cujos experimentos ou pesquisas para elicitar aversão ao risco deixam de ser hipotéticos e passam a incorporar aspectos financeiros reais.

A teoria tradicional de tomada de decisão sob incerteza assume que o grau de aversão ao risco relativa não varia com a escala dos retornos, sendo intrínseca e estável nos indivíduos (HARRISON; RUTSTRÖM, 2008). No entanto, algumas evidências não favorecem esse pressuposto quando se utilizam experimentos laboratoriais ou instrumentos de pesquisa que tentam mensurar a aversão ao risco (HOLT; LAURY, 2002; CUNHA, 2012; DICKHAUT et al., 2013). Nesse caso, a aferição incorreta do risco para indivíduos pode levar à correlação espúria de tal indicador com várias decisões de mercado envolvendo risco, precificações incorretas e prejuízos econômicos. Seguindo o exemplo do seguro, caso o intermediário financeiro tenha feito a elicitação de aversão ao risco por um mero experimento laboratorial hipotético, este pode estar ofertando um prêmio mais baixo do que o indivíduo estaria de fato disposto a pagar.

11 CONTEXTUS - Revista Contemporânea de Economia e Gestão. Especial 80 Anos FEAAC - 2018 
Este trabalho baseia-se na metodologia de Holt e Laury (2002) para extrair indicadores de aversão ao risco e testar a hipótese de que eles podem ser afetados por estímulos de magnitude em ganhos monetários hipotéticos ou reais. O estudo de Holt e Laury (2002) trouxe inovações importantes no campo dos experimentos laboratoriais, tais como a técnica de elicitação de aversão ao risco denominada Lista De Preços Múltiplos (Multiple Price Lists $M P L)$ : dada uma série ordenada de loterias pareadas, os indivíduos devem escolher uma de cada par e, dessa forma, revelar suas preferências. O padrão de escolhas do indivíduo determina seu tipo (amante, avesso ou neutro ao risco) e o seu nível de aversão ao risco. Essa metodologia foi adaptada em vários contextos, com diferentes propósitos e populações de interesse (LAURY, 2005; HOUSER et al., 2010; DICKHAUT et al., 2013; JACOBSON; PETRIE, 2009; CHARNESS; VICEISZA, 2011), sendo pouco utilizada em estudos de caso no Brasil. As exceções neste último caso são Cunha (2012) e Saurin et al. (2015), que aplicaram o experimento com alunos da Universidade Federal de Santa Catarina para investigar, respectivamente, o efeito incentivo e o modo como o nível de aversão ao risco pode influenciar decisões individuais de investimentos.

Este artigo, além de estimar o grau de aversão ao risco para um estudo de caso específico, também verifica os efeitos de escala e incentivo de forma integrada no mesmo experimento. A seção 2 abrange a revisão de literatura e o desenho do experimento, expondo a metodologia de elicitação de risco de Holt e Laury (2002) e aplicações que dali seguiram. Vale destacar que os participantes do experimento eram alunos da Faculdade de Economia, Administração, Atuária e Contábeis, da Universidade Federal do Ceará. A aplicação do experimento nesse tipo de população é padrão na literatura dado ele exigir certo grau de compreensão de si e do assunto (CHARNESS et al., 2013) ${ }^{1}$. A seção 3 discute a caracterização da amostra (seção 3.1), o problema de inconsistência das respostas (seção 3.2) e os resultados encontrados para a mensuração do risco e para os efeitos escala e incentivos (seção 3.3), que foram aferidos neste estudo de caso. Este debate é estendido nas conclusões, que detalha problemas e potenciais soluções do experimento.

\footnotetext{
${ }^{1}$ Discentes também foram utilizados na experiência de Holt e Laury (2002).
} 


\section{METODOLOGIAS DE ELICITAÇÃO DE RISCO E BASE DE DADOS}

\subsection{Elicitação de aversão ao risco}

As preferências de risco desempenham papel fundamental em alguns cenários econômicos como mercado de trabalho (ACEMOGLU; SHIMER, 1999; FALCO, 2014; HUIZEN; ALESSIE, 2016), mercado financeiro (DENUIT; EECKHOUDT, 2016; KRÄUSSL; LUCAS; SIEGMANN, 2012; PARDO, 2012), teoria dos jogos (TEYSSIER, 2012; LI et al. (2015); KIHLSTROM; ROTH; SCHMEIDLER, 1981; KIHLSTROM E ROTH, 1982), entre outros.

Diante da preferência dos indivíduos de assumirem mais ou menos risco, os tomadores de decisão são classificados como amantes ao risco, neutros ao risco e avessos ao risco. Para determinação empírica de como esses agentes se comportam diante do risco, estudos empíricos mensuram qual seria o grau de aversão ao risco desses indivíduos. A essa investigação denomina-se elicitação de aversão ao risco.

Entretanto, por vezes, assume-se, simplesmente, que os indivíduos são neutros ao risco ou apresentam aversão ao risco absoluta e/ou aversão ao risco relativa constante. Seja pela impossibilidade de obtenção de tal medida, seja pela conveniência computacional e matemática, tais facilidades provenientes destas suposições, ainda que plausíveis, irão condicionar as inferências resultantes (HARRISON; RUTSTRÖM, 2008). Para o adequado uso nas tomadas de decisão dos agentes, torna-se primordial que a elicitação do grau de aversão ao risco seja adequadamente determinada.

Neste sentido, economistas desenvolveram uma grande variedade de teorias e de metodologias para justificar e para mensurar preferências individuais de risco. Harrison e Rutström (2008) fazem uma abrangente revisão de experimentos para elicitação de aversão ao risco em ambientes controlados de laboratório. Charness et al. (2013), por sua vez, trazem em seu survey, a análise dos experimentos conforme o grau de complexidade dos diversos métodos.

Este trabalho utiliza a técnica de elicitação denominada Lista De Preços Múltiplos (Multiple Price Lists - MPL). Este método, apresenta uma série ordenada de loterias pareadas para que os indivíduos façam escolhas entre as mesmas. Os indivíduos devem escolher uma loteria para cada par de loterias apresentado e, desta forma, revelem suas preferências. A característica principal deste experimento é que o indivíduo só tem aumento nos seus retornos esperados se aceitar ou o aumento no risco ou na dispersão dos resultados, e pode-se, então, 
estimar uma medida de aversão ao risco com base no número de alternativas seguras selecionadas.

Um dos primeiros estudos que utilizou este método foi realizado por Miller, Meyer e Lanzetta (1969) onde os indivíduos foram confrontados com cinco loterias com valores esperados positivos iguais, mas com probabilidades de ganhar cada alternativa variáveis entre, $0,2,0,5$ e 0,8 . Os resultados mostram que quando a probabilidade de ganhar era aumentada, os indivíduos preferiam alternativas mais arriscadas - efeito foi atribuído às frequências relativa e absoluta de ganho. Outros estudos proeminentes foram feitos por Binswanger (BINSWANGER, 1980; BINSWANGER, 1981) que utilizaram este método para elicitar preferências de risco de fazendeiros da zona rural da Índia. O diferencial neste estudo, na época, foi que ele utilizou de retornos monetários reais a fim de incentivar os participantes a revelarem suas verdadeiras preferências. Outros estudos semelhantes foram realizados por Kahneman et al. (1990) para precificação de bens e efeito de patrimônio, Coller e Williams (1999) para elicitação de taxas de juros individuais (IDR - taxas nas quais os indivíduos estão dispostos a trocar o consumo presente pelo consumo futuro), Schubert et al. (1999) para elicitação de atitudes de risco específicas de gênero no mercado financeiro, Barr e Packard (2002) para elicitação de preferências de risco no contexto da seguridade social no Chile.

Mais recentemente o método foi popularizado pelos pesquisadores Charles A. Holt e Susan K. Laury. Holt e Laury (2002) realizaram um estudo cujo instrumento era uma lista com loterias pareadas. Em cada jogo, composto por um par de loterias, os indivíduos deveriam escolher entre a loteria mais segura (cujos retornos tinham menor variabilidade) e a loteria mais arriscada. $\mathrm{O}$ instrumento foi estruturado de maneira que o ponto de cruzamento de uma coluna para a outra pudesse ser utilizado para estimar o grau de aversão ao risco do indivíduo. A fim de determinar efeitos específicos, o experimento foi particionado em cinco tratamentos (escalas de valores), nos quais os retornos foram multiplicados por fatores de 20, 50 e 90 vezes o retorno inicial, mais baixo, e considerados hipotéticos em algumas etapas e reais em outras, onde o pagamento em dinheiro era feito de acordo com as alternativas escolhidas na etapa em questão. Embora os aumentos dos retornos não tenham efeito nas decisões dos indivíduos quando os jogos são hipotéticos, eles apresentam-se mais avessos ao risco quando os retornos são pagos de fato - indo contra a teoria de aversão ao risco relativa constante.

Desta maneira, o estudo de Holt e Laury (2002) trouxe inovações metodológicas importantes no campo dos experimentos laboratoriais que permitiu o estudo de um vasto 
conjunto de decisões para um melhor mapeamento da função utilidade, da forma funcional mais ampla e testável, e da verificação de efeito escala e efeito incentivo, que são grandes pressupostos da economia (pois os agentes reagem a incentivos).

Dando seguimento ao artigo original, Laury (2005) avalia se os indivíduos se comportam como se cada uma das escolhas envolvesse retornos certos ou se eles minimizam os retornos devido à seleção aleatória que é feita para realização dos pagamentos. De fato, o aumento do retorno tem efeito significativo nas escolhas seguras (aumento da aversão ao risco), se comparadas com o tratamento cujo retorno é o mais baixo, quando todas as dez decisões são pagas. Porém, não há diferença significativa entre pagar para apenas uma decisão ou para todas as dez quando o retorno é baixo - sugerindo que se pode utilizar um método de pagamento aleatório sem perda de qualidade dos dados, neste nível de retorno. Dickhaut et al. (2013) aprimoram esse estudo e mostram que se pode induzir as preferências dos indivíduos utilizando técnicas de recompensa de loterias binárias. O método (uma pontuação na primeira etapa convertida em chances de receber determinado valor monetário na segunda etapa) induz as preferências tais que as decisões em um ambiente cujas apostas são baixas podem refletir as escolhas feitas em um ambiente com apostas elevadas. Os resultados obtidos com esse método indicam que o comportamento dos indivíduos foi similar àquele apresentado pelos indivíduos nas etapas cujos pagamentos eram reais em Holt e Laury (2002).

A metodologia de Holt e Laury (2002) tem sido amplamente utilizada na literatura econômica de estudos empíricos de aversão ao risco. Harrison e Rutström (2008) e Charness et al. (2013) trazem um resumo dos principais trabalhos. No Brasil, pode-se citar Cunha (2012) e Saurin, et al. (2015).

Cunha (2012) restringe seu estudo para evidenciar a existência de diferentes níveis de aversão ao risco quando indivíduos são submetidos a incentivos de retornos monetários hipotéticos e retornos monetários reais em uma mesma escala. A amostra é alunos da Universidade Federal de Santa Catarina e do Instituo Federal de Santa Catarina. Os resultados apresentados não corresponderam às expectativas, tendo em vista que os testes com indivíduos de retornos hipotéticos apresentaram maior aversão ao risco do que testes com indivíduos recebendo retornos reais.

Já em Saurin et al. (2015), o uso da metodologia de Holt e Laury (2002) é utilizado como parte de um estudo da relação entre o viés do status quo, o perfil de risco e a habilidade quantitativa em estudantes de pós-graduação em Economia, Contabilidade e Gestão. Sua 
amostra engloba alunos de pós-graduação da universidade do Porto (Portugal) e da Universidade Federal de Santa Catarina.

Com outra metodologia para revelar o grau de disposição dos agentes em assumir risco no Brasil, deve-se citar também Tecles e Resende (2011) em sua investigação sobre a teoria dos Prospectos junto a alunos da Universidade de Brasília.

Neste artigo, o experimento de Holt e Laury (2002) é utilizado de modo a se investigar a disposição de alunos da Faculdade de Economia, Administração e Contabilidade da Universidade Federal do Ceará de assumirem risco. O mesmo se difere de Cunha (2012) e Saurin et al. (2015) pois utiliza duas loterias de escolha, em vez de um valor com certeza versus apenas uma loteria probabilística como ocorre nestes últimos artigos. Também se distingue de Holt e Laury (2002) no estudo de caso e nas escalas de testes adaptadas a moeda corrente, mas que mantiveram a mesma proporcionalidade de risco entre as escolhas. A seguir o desenho do experimento é ilustrado.

\subsection{Desenho do experimento}

O instrumento aplicado, reproduzido em apêndice, apresenta jogos compostos por duas loterias (opção A e opção B) com probabilidades de ganho variadas. O indivíduo deve fazer uma escolha entre as duas loterias pareadas, para cada um dos dez jogos listados, como exposto na Tabela 1. A última coluna com os valores esperados para cada jogo serve para indicar o ponto de inflexão das escolhas de um sujeito neutro ao risco para estes valores, não sendo apresentada ao indivíduo em momento algum.

De acordo com as escolhas dos indivíduos, as suas preferências são reveladas e podese estimar tanto o grau de aversão ao risco quanto à forma funcional da função utilidade. A fim de verificar hipóteses de efeito da escala e de efeito do incentivo monetário, além de validar as respostas a retornos mais elevados, o experimento foi conduzido sob condições hipotéticas e reais de pagamento. Os indivíduos fizeram as escolhas com base em retornos monetários mais baixos e mais altos, sendo a escala aumentada em 10,5 e 2,5 vezes o valor do retorno mais baixo. Note que os retornos para a opção A são menos variáveis dos que os retornos para a opção B. Dessa forma, considera-se a opção A como a opção mais segura e a opção B como a opção mais arriscada. 
No jogo 1, a chance de receber o maior valor é de $10 \%$ e a chance de receber o menor valor é de $90 \%$, em ambas as loterias. Nos jogos sucessivos, as chances de receber o valor maior vão aumentando e as chances de receber um valor menor vão diminuído, gradativamente. Assim, supõe-se que somente as pessoas muito amantes do risco optariam pela loteria B nos jogos iniciais. Um indivíduo neutro ao risco, escolheria a opção A nos quatro primeiros jogos e escolheria a opção B nos seis jogos seguintes, conforme os retornos esperados indicados na última coluna, que é dada por:

$$
\left(p_{1 A} * x_{1 A}+p_{2 A} * x_{2 A}\right)-\left(p_{1 B} * x_{1 B}+p_{2 B} * x_{2 B}\right)(1)
$$

Onde, $\mathrm{p}_{1 \mathrm{~A}}$ é a probabilidade de ocorrer o primeiro valor na loteria $\mathrm{A}$, $\mathrm{p}_{2 \mathrm{~A}}$ é a probabilidade de ocorrer o segundo valor na loteria $\mathrm{A}, \mathrm{p}_{1 \mathrm{~B}}$ é a probabilidade de ocorrer o primeiro valor na loteria $\mathrm{B}$, е р 2 в é a probabilidade de ocorrer o segundo valor na loteria $\mathrm{B}$. Estas probabilidades mudam ao longo dos jogos, mantendo-se constantes os respectivos valores correspondentes $\left(\mathrm{x}_{1 \mathrm{~A}}, \mathrm{x}_{2 \mathrm{~A}}, \mathrm{x}_{1 \mathrm{~B}} \text { e } \mathrm{x}_{2 \mathrm{~B}}\right)^{2}$.

A medida que a chance do retorno maior, $\mathrm{R} \$ 3,85$, neste caso, se torna suficientemente atraente para o indivíduo, ele deveria alternar as suas escolhas da loteria A, mais segura, para a loteria $\mathrm{B}$, mais arriscada. E, até aquelas pessoas mais avessas ao risco, deveriam optar pela opção B no jogo 0, visto que ela garante o maior valor com certeza.

Tabela 1 - Jogos e loterias apresentados no experimento

\begin{tabular}{c|c|c|c}
\hline JOGO & OPÇÃO A & OPÇÃO B & $\begin{array}{c}\text { E }(\mathbf{A})-\mathbf{E} \\
\text { (B) ] } \\
\text { RETORNO } \\
\text { ESPERADO }\end{array}$ \\
\hline 1 & $\begin{array}{c}1 / 10 \text { de } \mathrm{R} \$ 2,00 \text { e } 9 / 10 \text { de } \\
\mathrm{R} \$ 1,60\end{array}$ & $\begin{array}{c}1 / 10 \text { de } \mathrm{R} \$ 3,85 \text { e } 9 / 10 \text { de } \\
\mathrm{R} \$ 0,10\end{array}$ & $\mathrm{R} \$ 1,17$ \\
\hline 2 & $\begin{array}{c}2 / 10 \text { de } \mathrm{R} 2,00 \text { e } 8 / 10 \text { de } \\
\mathrm{R} \$ 1,60\end{array}$ & $\begin{array}{c}2 / 10 \text { de } \mathrm{R} \$ 3,85 \text { e } 8 / 10 \text { de } \\
\mathrm{R} \$ 0,10\end{array}$ & $\mathrm{R} \$ 0,83$ \\
\hline 3 & $\begin{array}{c}3 / 10 \text { de } \mathrm{R} \$ 2,00 \text { e } 7 / 10 \text { de } \\
\mathrm{R} \$ 1,60\end{array}$ & $\begin{array}{c}3 / 10 \text { de } \mathrm{R} \$ 3,85 \text { e } 7 / 10 \text { de } \\
\mathrm{R} \$ 0,10\end{array}$ & $\mathrm{R} \$ 0,50$ \\
\hline 4 & $\begin{array}{c}4 / 10 \text { de } \mathrm{R} \$ 2,00 \text { e } 6 / 10 \text { de } \\
\mathrm{R} \$ 1,60\end{array}$ & $\begin{array}{c}4 / 10 \text { de } \mathrm{R} \$ 3,85 \text { e } 6 / 10 \text { de } \\
\mathrm{R} \$ 0,10\end{array}$ & $\mathrm{R} \$ 0,16$ \\
\hline 5 & $\begin{array}{c}5 / 10 \text { de } \mathrm{R} 2,00 \text { e } 5 / 10 \text { de } \\
\mathrm{R} \$ 1,60\end{array}$ & $\begin{array}{c}5 / 10 \text { de } \mathrm{R} \$ 3,85 \text { e } 5 / 10 \text { de } \\
\mathrm{R} \$ 0,10\end{array}$ & $-\mathrm{R} \$ 0,18$ \\
\hline
\end{tabular}

(CONTINUA)

\footnotetext{
${ }^{2}$ Estes valores nos jogos da Tabela 1 são, respectivamente, R\$ 2,00, R\$ 1,60, R\$ 3,85 e R\$ 0,10.
} 


\begin{tabular}{c|c|c|c}
\multicolumn{2}{l}{ (CONTINUAÇÃO) } \\
\hline \multirow{2}{*}{6} & $\begin{array}{c}6 / 10 \text { de } \mathrm{R} \$ 2,00 \text { e } 4 / 10 \text { de } \\
\mathrm{R} \$ 1,60\end{array}$ & $\begin{array}{c}6 / 10 \text { de } \mathrm{R} \$ 3,85 \text { e } 4 / 10 \text { de } \\
\mathrm{R} \$ 0,10\end{array}$ & $-\mathrm{R} \$ 0,51$ \\
\hline \multirow{2}{*}{7} & $\begin{array}{c}7 / 10 \text { de } \mathrm{R} \$ 2,00 \text { e } 3 / 10 \text { de } \\
\mathrm{R} \$ 1,60\end{array}$ & $\begin{array}{c}7 / 10 \text { de } \mathrm{R} \$ 3,85 \text { e } 3 / 10 \text { de } \\
\mathrm{R} \$ 0,10\end{array}$ & $-\mathrm{R} \$ 0,85$ \\
\hline \multirow{2}{*}{8} & $\begin{array}{c}8 / 10 \text { de } \mathrm{R} 2,00 \text { e } 2 / 10 \text { de } \\
\mathrm{R} \$ 1,60\end{array}$ & $\begin{array}{c}8 / 10 \text { de } \mathrm{R} \$ 3,85 \text { e } 2 / 10 \text { de } \\
\mathrm{R} \$ 0,10\end{array}$ & $-\mathrm{R} \$ 1,18$ \\
\hline 9 & $\begin{array}{c}9 / 10 \text { de } \mathrm{R} \$ 2,00 \text { e } 1 / 10 \text { de } \\
\mathrm{R} \$ 1,60\end{array}$ & $\begin{array}{c}9 / 10 \text { de } \mathrm{R} \$ 3,85 \text { e } 1 / 10 \text { de } \\
\mathrm{R} \$ 0,10\end{array}$ & $-\mathrm{R} \$ 1,52$ \\
\hline \multirow{2}{*}{10} & $\begin{array}{c}10 / 10 \text { de } \mathrm{R} \$ 2,00 \text { e } 0 / 10 \text { de } \\
\mathrm{R} \$ 1,60\end{array}$ & $\begin{array}{c}10 / 10 \text { de } \mathrm{R} \$ 3,85 \text { e } 0 / 10 \text { de } \\
\mathrm{R} \$ 0,10\end{array}$ & $-\mathrm{R} \$ 1,85$ \\
\hline
\end{tabular}

Fonte: elaboração própria, adaptado de Holt e Laury (2002).

O experimento foi realizado em três etapas, detalhadas sequencialmente aos participantes. Inicialmente, antes do experimento, os indivíduos responderam um questionário com informações sócio-/sociodemográficas e de comportamentos de risco. A obtenção dessas informações tem o objetivo de descrever a amostra e contextualizar qualitativamente o estudo. Na primeira etapa, o procedimento de escolha é explicado e a tabela com os dez jogos e as duas opções de loterias é apresentada. Os indivíduos deveriam, então, fazer as escolhas que indicassem suas preferências ou pela loteria A ou pela loteria B para cada um dos dez jogos listados. É explicado que o experimento é hipotético, mas que, se fosse realizado, seria utilizado um dado de dez faces para a determinação dos valores ganhos - o dado seria lançado duas vezes, sendo o primeiro o sorteio do jogo e, depois, o sorteio do valor a ser recebido de acordo com a opção escolhida previamente. Esta etapa é igual para todos os grupos e, além de ser um treinamento, esta etapa serve de controle para o experimento. $\mathrm{Na}$ etapa dois, o experimento mantem-se hipotético e o procedimento de escolha deve ser feito da mesma maneira da etapa 1, com o diferencial de que a escala de valores utilizada varia entre 10,5 e 2,5 vezes os valores apresentados na etapa inicial, como descrito na Tabela $2 \mathrm{Na}$ terceira e última etapa, o experimento deixa de ser hipotético, a premiação em dinheiro é divulgada, alguns indivíduos seriam sorteados (com probabilidade definida arbitrariamente conforme o tamanho da turma) para jogar e receberiam o valor monetário, de acordo com as preferências indicadas nesta última etapa do experimento, independentemente do que haviam marcado nas etapas anteriores. Os valores apresentados nesta etapa, novamente, variam em escala de 10,5, 2,5 e 1 vez o valor da escala original. 
Tabela 2 - Escalas e valores do experimento

\begin{tabular}{c|cc|cc}
\hline Escala & \multicolumn{3}{|c|}{ Loteria A } & \multicolumn{2}{c}{ Loteria B } \\
\hline $1 \mathrm{x}$ & $\mathrm{R} \$ 2,00$ & $\mathrm{R} \$ 1,60$ & $\mathrm{R} \$ 3,85$ & $\mathrm{R} \$ 0,10$ \\
$2,5 \mathrm{x}$ & $\mathrm{R} \$ 5,00$ & $\mathrm{R} \$ 4,00$ & $\mathrm{R} \$ 9,60$ & $\mathrm{R} \$ 0,25$ \\
$5 \mathrm{x}$ & $\mathrm{R} \$ 10,00$ & $\mathrm{R} \$ 8,00$ & $\mathrm{R} \$ 19,25$ & $\mathrm{R} \$ 0,50$ \\
$10 \mathrm{x}$ & $\mathrm{R} \$ 20,00$ & $\mathrm{R} \$ 16,00$ & $\mathrm{R} \$ 38,50$ & $\mathrm{R} \$ 1,00$ \\
\hline \multicolumn{3}{c}{ Fonte: elaboração própria. }
\end{tabular}

O experimento sempre foi conduzido na mesma ordem, sendo, em resumo, a etapa inicial com valores hipotéticos mais baixos, a etapa intermediária com valores hipotéticos variando em escala dos valores iniciais, e a última etapa com possibilidade de ganhos reais e valores variando em escala dos valores iniciais.

Assim, pode-se avaliar o efeito do aumento da escala de valores da etapa um para a etapa dois. E, quando as etapas dois e três apresentam os mesmos valores, pode-se avaliar o efeito dos incentivos na decisão das pessoas.

Independentemente da escala de valores adotada no instrumento, pode-se utilizar o mesmo conceito de número de escolhas seguras para se obter estimativas de grau de aversão ao risco dos indivíduos. Se o número de escolhas seguras do indivíduo for igual a 4, este indivíduo será neutro ao risco, menor que quatro será amante ao risco e acima de quatro avesso ao risco. Quanto maior for o número de escolhas seguras maior será o seu nível de aversão ao risco.

Para um indivíduo com aversão ao risco constante, a multiplicação de todos os valores das loterias por um fator $k$ qualquer não deveria alterar o processo de decisão do mesmo. Quando isto ocorre observamos um efeito escala, cuja regularidade de ocorrência pode ser verificada (ou não) com um teste de diferenciais de médias ${ }^{3}$ para o número de escolhas seguras entre as etapas 1 e 2. Da mesma forma, um indivíduo não deveria mudar suas escolhas em um mesmo experimento para duas loterias de mesma magnitude apenas pelo fato de uma ser hipotética e a outra ser real. Isso evidencia um efeito incentivo, que também pode ser averiguado por um teste de diferenciais de médias entre a segunda e a terceira etapas.

\footnotetext{
${ }^{3}$ Teste t para amostras pareadas. No teste empírico, será utilizado o comando “ttest” do software Stata versão 13.
} 
Tabela 3 - Descrição dos experimentos por turma

\begin{tabular}{cccccc}
\hline & & & \multicolumn{3}{c}{ Escala de valores } \\
\cline { 4 - 6 } turma & n & p_sorteio & etapa_1 & etapa_2 & etapa_3 \\
\hline 1 & 14 & 1 & 1 & 2,5 & 1 \\
2 & 16 & 0,50 & 1 & 2,5 & 2,5 \\
3 & 19 & 0,79 & 1 & 5 & 5 \\
4 & 27 & 0,56 & 1 & 10 & 1 \\
5 & 26 & 0,58 & 1 & 10 & 10 \\
6 & 13 & 0,15 & 1 & 10 & 10 \\
7 & 15 & 0,13 & 1 & 10 & 10 \\
8 & 30 & 0,13 & 1 & 2,5 & 10 \\
\hline \multicolumn{5}{c}{}
\end{tabular}

Os participantes deste experimento são alunos de graduação dos cursos de Finanças, Administração, Contábeis e Economia e alunos de pós-graduação do Mestrado Profissional em Economia do Setor Público da Universidade Federal do Ceará, presentes em turmas selecionadas de forma a evitar múltipla participação no experimento. No total, foram 160 indivíduos participantes distribuídos em 8 turmas com número variado de alunos. A Tabela 3 mostra a metodologia adotada, com as escalas de valores adotadas em cada etapa e turma, a probabilidade de o aluno ser sorteado na turma no fim do experimento para jogar a loteria ${ }^{4}$, e o número de alunos em cada turma.

\section{RESULTADOS}

\subsection{Caracterização da amostra}

Dos 160 alunos entrevistados 56\% eram homens. A idade dos entrevistados varia entre 17 e 67 anos, com média de 23 anos (dp = 4,784) na graduação e de 43 anos (dp = 10,413) na pós-graduação. A raça predominante é parda (53,75\%), seguida por brancos $(35,63 \%)$, negros $(8,12 \%)$ e outras $(2,50 \%)$. Os solteiros formam $71,24 \%$ da amostra, os casados $25,63 \%$ e os separados/divorciados 3,13\%. Em relação à família, 43,31\% possuem até três membros, $47,14 \%$ possuem quatro ou cinco membros e o restante, 9,55\%, possuem de seis a oito membros. Ainda em relação à família, 65,60\% declaram-se filhos, 16,56\% são cônjuges,

\footnotetext{
${ }^{4}$ Por restrições orçamentárias, nem todos poderiam jogar a loteria real na etapa 3. Então optou-se por realizar um sorteio de alguns alunos. Essa informação era dada previamente ao início desta etapa.
} 
15,29\% são chefes e 2,55\% ocupam outra posição familiar. Aproximadamente $43 \%$ deles estudaram o ensino médio em escola pública e $32 \%$ tem os estudos da faculdade como única atividade. Todos os alunos da pós-graduação relataram renda maior que cinco salários mínimos, sendo que $88 \%$ deles relataram renda maior que dez salários mínimos. Já entre os alunos da graduação, 16,67\% declarou renda menor que dois salários mínimos, 37,25\% entre dois e três, $33,34 \%$ entre três e dez, e apenas $12,75 \%$ declarou renda maior que dez salários mínimos.

Quando perguntados se já haviam sofrido, alguma vez, um assalto, $81 \%$ disse que sim. Destes, $28 \%$ declararam que o evento ocorreu há menos de um ano e $8 \%$ que o evento foi violento. Quando questionados se eles dirigiriam um veículo depois de ingerir bebida alcoólica, 22,5\% disse não ter carteira de habilitação, mas 26,25\% disse que isso já aconteceu ou que acontece eventualmente. Entre aqueles indivíduos (77,50\%) que já receberam um pedido de empréstimo de dinheiro por parte de um parente ou de um amigo, 37,10\% disse não emprestariam de maneira nenhuma, 10,48\% não voltariam a emprestar, 29,84\% emprestariam, e $22,58 \%$ emprestariam novamente.

Como estas características não foram homogêneas entre as turmas os testes de efeito escala e efeito incentivo devem ser realizados agregando o maior número de turmas possíveis que jogam as mesmas loterias em duas ocasiões. Isto não apenas confere uma comparação adequada, como oferece uma maior robustez estatística. No entanto, antes de realizar estes testes é preciso checar a consistência das respostas proferidas pelos alunos.

\subsection{Inconsistências}

O grau de aversão ao risco de um indivíduo pode ser estimado pelo número de jogos em que ele optou pela loteria A (a opção mais segura), ou seja, aquela que apresenta menor amplitude entre os valores propostos. A consistência das respostas se dá pelo padrão das opções marcadas e implica, obrigatoriamente, a não alternância entre as colunas ao longo dos jogos da mesma etapa e a marcação da opção B no último jogo.

Em toda a amostra, independentemente da etapa, da escala e do incentivo oferecido, 25 pessoas foram inconsistentes na escolha da loteria no jogo 10 - aquele jogo cuja loteria $\mathrm{B}$ pagaria o maior valor com certeza - e optaram pela loteria A. Dos 160 indivíduos, 16 escolheram a opção A do jogo 0 na etapa 1,15 na etapa 2 e apenas 12 na última etapa. Essa redução pode sugerir que houve um processo de aprendizado das regras do jogo ao longo das 
etapas e/ou um aumento do interesse pessoal no incentivo oferecido, visto que os valores eram maiores na etapa dois e eram pagos, de fato, na etapa três, logo, aumento do grau de aversão ao risco.

Na etapa $1,73 \%$ pessoas apresentaram um padrão de resposta racional coerente, ou seja, com nenhum ou apenas um ponto de inflexão no seu padrão de resposta. A distribuição de frequências das repostas sugere algum grau de indecisão nos jogos cujas probabilidades são mais semelhantes (jogos 4, 5 e 6). Supõe-se, ainda, que uma parcela dos indivíduos realizou o experimento com grau considerável de descomprometimento, alternando as opções diversas vezes. Na primeira etapa, $26 \%$ das pessoas mostraram cinco ou mais pontos de inflexão no padrão de resposta. Na etapa dois, este percentual reduziu para $10 \%$ e manteve-se na última etapa. O percentual de padrão de resposta coerente aumentou nas etapas seguintes. Na etapa dois, foram $80 \%$ e, na etapa três, foram $85 \%$ dos indivíduos que alternaram suas respostas da opção A para a opção B no máximo uma única vez.

Os indivíduos com respostas inconsistentes foram retirados da amostra com um número final de 108 alunos o que implica uma taxa de inconsistência bastante alta de 32,5\%, quando comparada a taxa de 13,2\% encontrada em Holt e Laury (2002) $)^{5}$, e 9\% em Cunha (2012). Ainda é menor que as taxas de 55\% encontradas em Jacobson e Petrie (2009) e 75\% em Charness e Viceisza (2011), mas vale destacar que a população pesquisada nestes últimos artigos foram respectivamente de adultos em Ruanda e de agricultores rurais em Senegal.

\subsection{Comparação de grupos, efeito escala e efeito incentivo}

A Tabela 4 a seguir mostra o número médio de escolhas da loteria segura (loteria $\mathrm{A}$ ) por turmas, etapas e escalas, após a exclusão das respostas inconsistentes. A última linha desta tabela mostra uma média geral para cada etapa e escala de valor das loterias jogadas, sendo um primeiro indicador de aversão ao risco.

Vale destacar que os testes de média para todos os indicadores mostraram que os mesmos eram estatisticamente diferentes de 4, que seria o valor para definir um indivíduo neutro ao risco. Neste caso os valores variaram de 4,786 na loteria 1x da Etapa 3 a 5,847 na

\footnotetext{
${ }^{5}$ Percentual apenas para os que alternaram respostas.
} 
loteria de escala 10x também desta etapa. O primeiro valor é um pouco menor que o encontrado em Houser et al. (2010) que encontrou um média de 5,860 para uma loteria proporcional em escala mas medida em euros. Holt e Layry (2002) encontraram uma amplitude de valores maiores (entre 4,800 e 7,200), mas vale destacar que as escalas de valores foram bem mais altas $^{6}$. Portanto, em termos absolutos, mostrou-se neste estudo de caso uma população avessa e sensível ao risco como também encontrado em outros estudos de casos com mesmo tipo de população.

Tabela 4 - Médias de escolhas seguras em cada etapa, descrição por turma

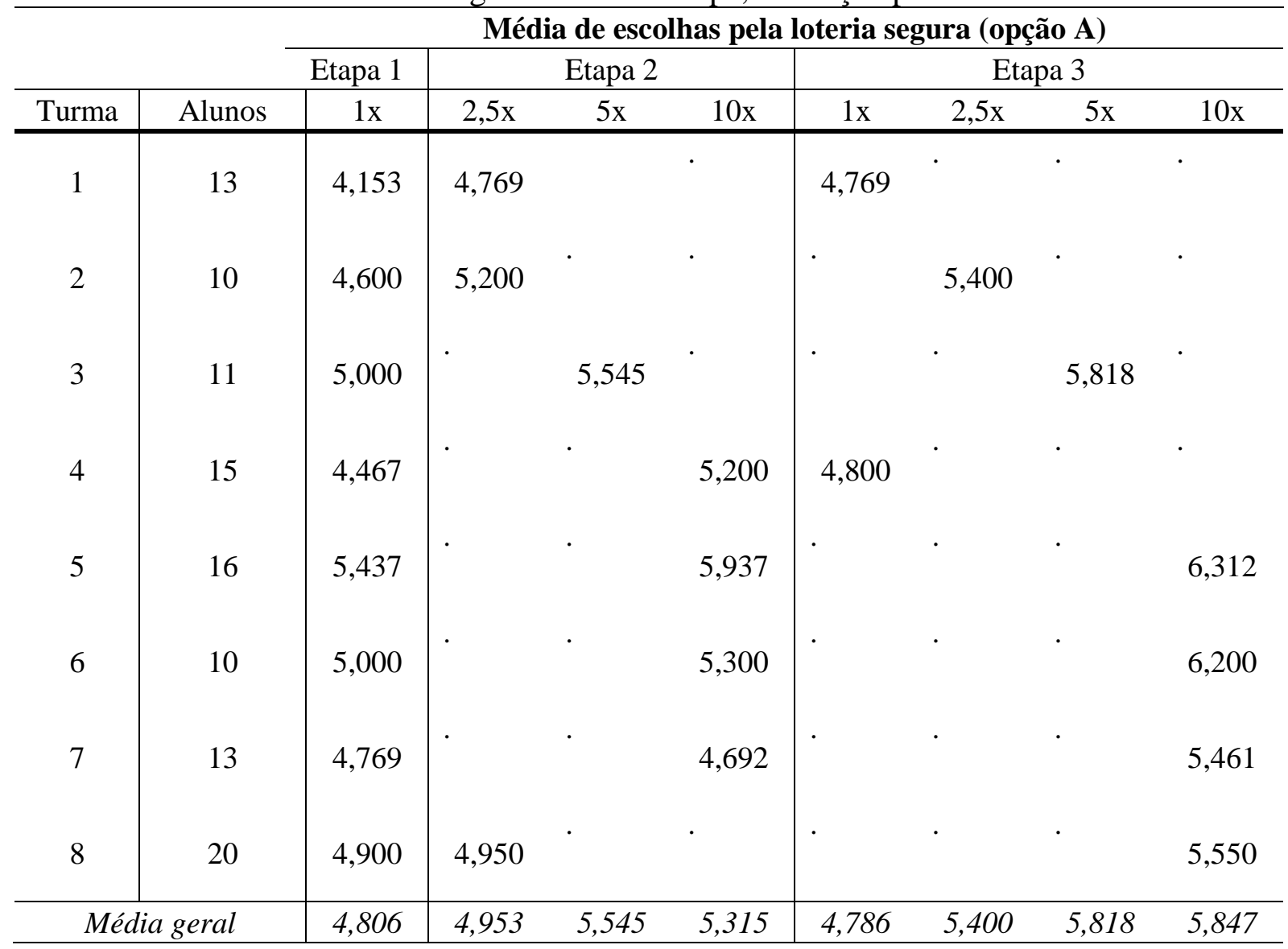

Fonte: elaboração própria.

Nota-se ainda na Tabela 4 uma tendência de aumento no valor deste indicador quando se passa da primeira para a segunda etapa (aumento de escala), e da segunda para terceira (efeito incentivo) mantendo-se a mesma escala de loterias. Isto pode ser observado tanto para a média geral como para cada turma em isolado. As exceções em termos de turmas foram a turma 1 que manteve o mesmo valor de média entre a segunda e a terceira etapas, e a turma quatro que diminuiu o número médio de escolhas seguras também entre estas etapas. No entanto, vale

\footnotetext{
${ }^{6}$ As escalas de referência em Holt e Layry (2002) foram de 20x, 50x e 90x.
} 
destacar que as escalas de medidas foram diferentes sendo a da etapa 3 menor que a da etapa 1 , mesmo esta última sendo paga.

Para se testar as hipóteses de efeito escala e efeito incentivos as médias de escolhas seguras foram calculadas para o conjunto de turmas que fizeram exatamente os mesmos jogos entre as etapas 1 e 2 (efeito escala) e entre as etapas 2 e 3 . Os resultados estão reportados na Tabela 5 que mostra a média de escolhas certas por grupos de turmas e as loterias comparadas em cada teste. São duas possibilidades de efeito escala. A primeira quando a mudança de escala na segunda etapa é baixa, passando de $1 \mathrm{x}$ na primeira para $2,5 \mathrm{x}$ na segunda, incluindo alunos das turmas 1,2 e 8. Neste caso, observa-se que a média de escolhas seguras aumenta entre etapas mas o diferencial entre as mesmas $(0,348)$ não é estatisticamente significante.

$\mathrm{Na}$ segunda possibilidade de efeito escala compara-se a possibilidade de ganhos hipotéticos ao se passar da escala de 1x na primeira etapa para a escala de 10x na segunda, envolvendo os alunos das turmas 4, 5, 6 e 7. A média de escolhas seguras passa de 4,926 na primeira etapa de valor mais baixo para 5,314 na segunda de valor mais alto, sendo o diferencial de 0,388 estatisticamente significante. Desta forma, evidencia-se um efeito escala, mas apenas quando a mudança na mesma é de magnitude considerável. Tal resultado é diferente de Holt e Laury (2002) que não encontraram evidência de efeito escala quando os pagamentos eram hipotéticos, mesmo sendo as escalas utilizadas ainda maiores naquele estudo.

Tabela 5 - Testes de Efeito Escala e Efeito Incentivo

\begin{tabular}{|c|c|c|c|c|}
\hline \multirow[b]{3}{*}{ Turmas } & \multicolumn{4}{|c|}{ Média de escolhas pela loteria segura (opção A) } \\
\hline & \multicolumn{4}{|c|}{ Efeito Escala } \\
\hline & Loterias Comparadas & Etapa 1 & Etapa 2 & Diferença \\
\hline 1,2 e 8 & $\begin{array}{l}\text { 1x Etapa1 } \\
\text { Versus } \\
\text { 2,5x Etapa } 2\end{array}$ & $\begin{array}{l}4,605 \\
(1,62)\end{array}$ & $\begin{array}{l}4,953 \\
(1,44)\end{array}$ & $\begin{array}{l}0,348 \\
(1,55)\end{array}$ \\
\hline \multirow[t]{2}{*}{$4,5,6$ e 7} & $\begin{array}{c}1 \times \text { Etapa1 } \\
\text { Versus } \\
10 \text { x Etapa } 2\end{array}$ & $\begin{array}{l}4,926 \\
(1,42)\end{array}$ & $\begin{array}{l}5,314 \\
(1,51)\end{array}$ & $\begin{array}{c}0,388^{*} \\
(0,03)\end{array}$ \\
\hline & \multicolumn{4}{|c|}{ Efeito Incentivo } \\
\hline Turmas & Loterias Comparadas & Etapa 2 & Etapa 3 & Diferença \\
\hline $\begin{array}{c}2,3,5,6 \mathrm{e} \\
7\end{array}$ & $\begin{array}{c}(2,5 x, 5 x, \text { e } 10 x) \text { Etapa } 2 \\
\text { Versus } \\
(2,5 x, 5 x, \text { e } 10 x) \text { Etapa } 3\end{array}$ & $\begin{array}{l}5,366 \\
(1,51)\end{array}$ & $\begin{array}{l}5,866 \\
(1,77)\end{array}$ & $\begin{array}{c}0,500^{*} \\
(1,41)\end{array}$ \\
\hline
\end{tabular}

Fonte: elaboração própria. Desvio-padrão entre parênteses.

*Diferença estatisticamente significante ao nível de $95 \%$ de confiança. 
Com relação ao efeito incentivo, as médias de escolhas seguras foram comparadas entre as etapas 2 (hipotética) e 3 (pagamento real) para os alunos que pertenciam as turmas 2, 3, 5, 6 e 7, que não modificaram suas respectivas escalas de valores entre estas etapas. Percebe-se na parte debaixo da Tabela 5, que o grau de aversão ao risco medido pela média de escolhas seguras passa de 5,366 para 5,866 quando o pagamento real é anunciado ao início da última etapa do experimento. A diferença de 0,5 pontos entre as médias é estatisticamente significante e maior pontualmente que a encontrada no efeito escala. Este resultado qualitativo equivale ao encontrado em Holt e Laury (2002) mas difere do encontrado em Cunha (2012), que encontrou uma aversão ao risco maior quando os pagamentos eram hipotéticos.

Uma outra forma de analisar os efeitos escala e incentivo é a comparação gráfica da proporção de escolhas seguras para cada jogo nas diferentes etapas (e escalas) de valores como mostra o Gráfico 1 abaixo. Ao longo dos 10 jogos de cada etapa um indivíduo neutro ao risco hipotético (trajetória laranja com traço) escolheria a loteria segura nas quatro primeiras e depois mudaria para opção B nos jogos seguintes (de 5 a 10). A fração média neste caso seria de 0,4 (4 escolhas seguras de 10 possíveis).

Percebe-se que o padrão dos resultados empíricos encontrados se diferencia da neutralidade, e a medida que as trajetórias vão se deslocando para direita aumenta o grau de aversão ao risco. Isto ocorre quando se passa de uma loteria básica da primeira etapa $(1 \mathrm{x}-$ trajetória azul com triângulo) para uma escala maior e hipotética na segunda etapa (10x trajetória cinza com quadrado), e para uma com pagamentos efetuados (10x - trajetória amarela com bolinhas).

Observa-se que a média das frações nos dez jogos para cada trajetória aumenta sinalizando um incremento na aversão ao risco. O percentual de indivíduos que escolhem mais de quatro jogos com escolhas seguras aumenta de 55,9\% na loteria básica, para 70,3\% na loteria de 10x hipotética e para $81,7 \%$ na loteria paga, reforçando tanto o efeito escala como o efeito incentivo. Estes últimos percentuais de indivíduos avessos ao risco não se diferem muito dos encontrados em outros experimentos, como em Saurin et al. (2010) e Houser et al. (2010) que encontraram taxas de $73,6 \%$ e $76 \%$, respectivamente, sendo que o primeiro possuía uma escala de loteria bem mais alta e o segundo a mais baixa de todas $(1 \mathrm{x})$. 
Gráfico 1 - Trajetória da fração de escolhas seguras por tipo de jogo

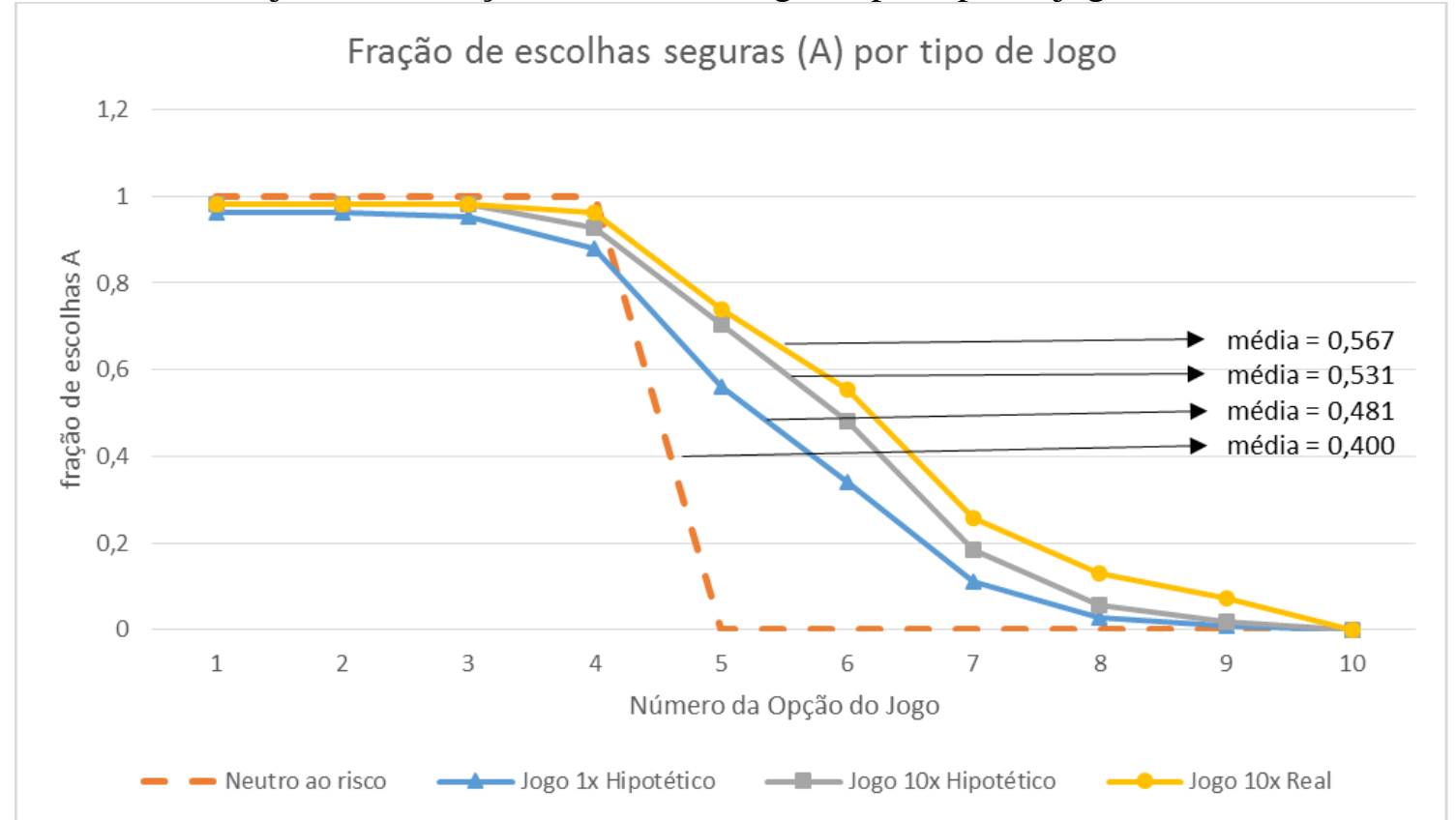

Fonte: elaboração própria.

Outra estatística importante de análise dos efeitos escala e incentivos ressalta por Holt e Laury (2002) é o percentual de indivíduos que mudam o número de opções seguras escolhidas entre etapas. Dos discentes das turmas 4, 5, 6 e7, 37\% deles mudaram o número de escolhas seguras entre as etapas 1 e 2; das turmas 2, 3, 5, 6 e 7, 40\% deles mudaram seus níveis de aversão ao risco entre as etapas 2 e 3 , ratificando os efeitos escala e incentivo respectivamente.

Estes últimos resultados deixam evidente que o processo de elicitação de risco dos indivíduos demanda um esforço metodológico e financeiro por parte do agente interessado, principalmente se o mesmo utilizar loterias pareadas ou escolhas de opções monetárias envolvendo riscos. Neste estudo de caso, mostrou-se que a falta de escalonamento nas opções e incentivo real deixaria uma boa parte dos indivíduos com um nível de aversão ao risco bastante subdimensionada. Se esta medida de aversão for parâmetro importante para precificação (apólice de seguros, por exemplo), este viés pode levar a prejuízos econômicos consideráveis. 
Este artigo estimou indicadores de aversão ao risco para indivíduos seguindo a metodologia de elicitação de risco com loterias, proposta por Holt e Laury (2002), que se tornou um importante marco referencial. O estudo de caso teve como população alvo discentes da Faculdade de Economia, Administração, Atuária e Contabilidade da Universidade Federal do Ceará que foram classificados de maneira geral como avessos ao risco, de magnitude moderada quando comparados com outras amostras específicas de discentes.

Além da constatação de os indivíduos entrevistados não serem neutros ao risco, verificou-se também que as estimativas dos níveis de aversão ao risco podem variar conforme os valores contidos nas loterias (efeito escala) e a efetivação de pagamento por participação no experimento (efeito incentivo). Experimentos que não consideram essas possibilidades podem estar subestimando o nível de aversão ao risco dos indivíduos, e isso pode ter algumas implicações metodológicas e econômicas.

Se o indicador do nível de aversão ao risco dos indivíduos for utilizado como variável determinante de outras decisões econômicas em modelos de regressão ou correlação, por exemplo, as estimativas de efeitos podem se tornar enviesadas no caso de loterias hipotéticas, uma vez que alguns indivíduos podem não estar revelando seu nível real de aversão ao risco. Nesse caso, pode-se sugerir a combinação de loterias com outros instrumentos de elicitação de aversão ao risco, como realizado em Saurin et al. (2015) para gerar indicadores múltiplos, ou modelos com controles para efeitos individuais ${ }^{7}$.

No caso em que a medição do nível de aversão ao risco é fato classificatório dos indivíduos, para os quais podem ser ofertados produtos envolvendo risco como empréstimos bancários ou seguros, um viés de estimação pode representar um pareamento inconsistente entre os preços e os riscos subjacentes assumidos. A subestimação dos riscos pode levar a precificações menores, capazes de levar a sinistros e insolvências acima do planejado pelas operadoras de seguro ou instituições de financiamento. Dada a importância da mensuração do grau de aversão ao risco neste caso, o desenho do processo e dos instrumentos de elicitação devem ser bem dimensionados em escalas e incentivos.

\footnotetext{
${ }^{7}$ Modelos de painel ou multinível com efeitos fixos, por exemplo.
} 
Um ponto adicional importante para o estudo de caso foi a observação de um percentual alto de respostas inconsistentes encontradas entre os participantes do experimento. Mais de $30 \%$ dos discentes mostraram algum tipo de inconsistência nas suas respostas, seja por desinteresse pelo experimento, seja pelo não entendimento do instrumento etc. Esse percentual foi bem mais alto que o encontrado em estudos similares como o de Cunha (2012) (9\%) e de Holt e Laury (13\%). É possível que a falta da cultura de pesquisa e do conhecimento da economia experimental possam estar contribuindo para esse nível elevado de descompromisso. A utilização de laboratórios específicos para o experimento e uma melhor preparação dos alunos em voluntariado também podem ser instrumentos importantes para a melhor qualificação da pesquisa.

\section{REFERÊNCIAS}

ACEMOGLU, Daron; SHIMER, Robert. Efficient unemployment insurance. Journal of Political Economy, v. 107, n 5, p. 893-928, 1999.

BARR, Abigail; PACKARD, Truman. Revealed Preference and self-insurance: can we learn from the self-employed in Chile? Policy Research Working Paper, Jan. 2002, World Bank, Washington DC.

BINSWANGER, Hans. Attitudes toward risk: experimental measure in rural India. American Journal of Agricultural Economics, v. 62, n. 3, p. 395-407, 1980.

Attitudes toward risk: theoretical implications of an experiment in rural India. Economic Journal, v. 91, n. 364, p. 867-890, 1981.

CHARNESS, Gary; VICEISZA, Angelino. Comprehension and risk elicitation in the field: evidence from rural Senegal. Mimeo. 2011. Disponível em: <http://excen.gsu.edu/fur2012/fullpapers/aviceisza.pdf〉. Acesso em: 13 set. 2018.

CHARNESS, Gary; GNEEZY, Uri; IMAS, Alex. Experimental methods: Eliciting risk preferences. Journal of Economic Behavior and Organization, v. 87, p. 43-51, 2013.

COLLER, Maribeth; WILLIAMS, Melonie. Eliciting individual discount rates. Experimental Economics, v. 2, n. 2, p. 107-127, 1999. 
CUNHA, Vitor. Aversão ao risco e incentivos: uma análise experimental. Monografia (Curso de Ciências Econômicas) - Universidade Federal de Santa Cantarina, 2015.

DICKHAUT, John; HOUSER, Daniel; AIMONE, Jason; TILA, Dorina; JOHNSON, Cathleen. High stakes behavior with low payoffs: inducing preferences with holt-laury gambles. Journal of Economic Behavior \& Organization, v. 94, p. 183-189, 2013.

DENUIT, Michel; EECKHOUDT, Louis. Risk aversion, prudence, and asset allocation: a review and some new developments. Theory and Decision, v. 80, n. 2, p. 227-243, 2016.

FALCO, Paolo. Does risk matter for occupational choices? Experimental evidence from an African labour market. Labour Economics, v. 28, p. 96-109, 2014.

HAUSER, Daniel; SCHUNK, Daniel; WINTER, Joachim. Distinguishing Trust from Risk: An anatomy of the investment game. Journal of Economic Behavior \& Organization, v. 74, n. 1-2, p. 72-81, 2010.

HARRISON, Glenn. Maximum likelihood estimation of utility functions using Stata. University of Central Florida, Working Paper 06-12. 2008. Disponível em: $<$ https://www.researchgate.net/publication/228785881_Maximum_likelihood_estimation_of_ utility_functions_using_Stata>. Acesso em: 14 mar. 2017.

HARRISON, Glenn; RUTSTRÖM, Elisbet. Risk Aversion in the laboratory. Research in Experimental Economics, v. 12, p. 41-196, 2008.

HOLT, Charles; LAURY, Susan. Risk aversion and incentive effects. American Economic Review, v. 92, n. 5, p. 1644-1655, 2002.

JACOBSON, Sarah; PETRIE, Ragan. Learning from mistakes: what do inconsistent choices over risk tell us? Journal of Risk and Uncertainty, v. 38, n. 2, p. 143-158, 2009.

KAHNEMAN, Daniel; KNETSCH, Jack; THALER, Richard. Experimental tests of the endowment effect and the Coase theorem. The Journal of Political Economy, v. 98, n. 6, p. 1325-1348, 1990.

KIHLSTROM, Richard. E.; ROTH Alvin. Risk aversion and the negotiation of insurance contracts. Journal of Risk and Insurance. v. 49, p. 372-387, 1982. 
; SCHMEIDLER, David. Risk aversion and solutions to Nash's bargaining problem. In: MOESCHLIN, Otto; PALLASCHKE, Diethard (Orgs). Game Theory and Mathematical Economics, Ed. Amsterdam: North-Holland Publishing Company, 1981. p. 65-71.

KRÄUSSL, Roman; LUCAS, Andre; SIEGMANN, Argen. Risk aversion under preference uncertainty. Finance Research Letters, v. 9, n. 1, p. 1-7, 2012.

LAURY, Susan. Pay one or pay all: random selection of one choice for payment. Andrew Young School of Policy Studies Research Paper Series, n. 06-13. 2005. Disponível em: <http://dx.doi.org/10.2139/ssrn.894271>. Acesso em: 142017.

LI, Sanxi; SUN, Hailin; YAN, Jianye; YIN, Xundong. Risk aversion in the Nash bargaining problem with uncertainty. Journal of Economics. v. 115, n. 3, p. 257-274, 2015.

LUCE, Duncan. Individual choice behavior. Ed. New York: Wiley, 1959.

MILLER, Louis; MEYER, David; LANZETTA, John. Choice among equal expected value alternatives: sequential effects of winning probability level on risk preferences. Journal of Experimental Psychology, v. 79, n 3, p. 419-423, 1969.

PARDO, Cristian. Risk aversion and business cycles: an empirical analysis. The Quarterly Review of Economics and Finances, v. 52, p. 413-426, 2012.

SAURIN, Valter; VAREJÃO, José; COSTA JR., Nilton; PRATES, Wlademir. Estudo Comparativo do viés do status quo e perfil de risco em tomadas de decisões por estudantes de cursos de pós-graduação. RAM - Revista de Administração Mackenzie, v. 16, n. 5, p. 95$126,2015$.

SCHUBERT, Renate; BROWN, Martin; GYSLER, Matthias; BRACHINGER, Hans. Financial decision-making: are women really more risk averse? American Economic Review (Papers \& Proceedings), v. 89, n 2, p. 381-385, 1999.

TECLES, Patrícia; RESENDE, José. A simple method for eliciting preference when exposed to risk: a case study using lottery simulation. Brazilian Review of Econometrics, v. 31, n 2, p. 201-229, 2011

TEYSSIER, Sabrina. Inequity and risk aversion in sequential public good games. Public Choice, v. 151, n. 1, p. 91-119, 2012. 
VAN HUIZEN, Thomas; ALESSIE, Rob. Risk Aversion and Job Mobility. Working Papers, n. 16-09, Utrecht School of Economics, 2016.

WILCOX, Nathanael. Stochastic models for a binary discrete choice under risk: a critical primer and econometric comparison. In: COX, James; HARRISON, Glenn (Org.). Risk aversion in experiments. Research in Experimental Economics, v. 12. UK: Emerald, 2008. p. 197-292. 


\section{APÊNDICE}

\section{Folha de informações complementares}

Este experimento faz parte de uma pesquisa sobre Análise de Risco da minha tese de doutorado em Economia - CAEN/UFC. Suas respostas serão consideradas confidenciais, e é fundamental que elas sejam sérias e verdadeiras. A sua participação é voluntária e de grande importância para mim!

O experimento se dará em três etapas.

Preencha seus dados, por gentileza:

\section{Curso:}

Nascimento (mês/ano):

\section{Matrícula:}

Raç:
( ) branca
( ) negra

Gênero:

( ) $\mathrm{M}$

( ) parda

( ) amarela

( ) F

Ensino médio:

( ) escola pública

( ) escola particular

Situação estudantil: ( ) estuda apenas

( ) estuda e trabalha

Situação de emprego/estágio/bolsa:
( ) não tem e não procura
( ) não tem e procura
( ) meio turno
( ) turno integral
$\begin{array}{lllll}\text { Estado civil: } & \text { ( ) solteiro } & \text { ( ) casado } & \text { ( ) separado/divorciado } & \text { ( ) viúvo } \\ \text { Posição na família: } & \text { ( ) chefe } & \text { ( ) cônjuge } & \text { ( ) filho/enteado } & \text { ( ) outro }\end{array}$

$N^{0}$ de membros na família:

Renda familiar em salários mínimos (sm):
( ) não sabe
( ) até $1 \mathrm{sm}(\mathrm{R} \$ 880,00)$
( ) prefere não responder
( ) de 2 a 3 sm ( R \$ 1.760,00 - R \$2.640,00)
( ) de 1 a 2 sm $(\mathrm{R} \$ 880,00-\mathrm{R} \$ 1.760,00)$
( ) de 5 a $10 \mathrm{sm}(\mathrm{R} \$ 4.400,00-\mathrm{R} \$ 8.800,00)$
( ) de 3 a $5 \mathrm{sm}(\mathrm{R} \$ 2.640,00-\mathrm{R} \$ 4.400,00)$
( ) mais de $10 \mathrm{sm}(\mathrm{R} \$ 8.800,00)$

Já sofreu assalto/roubo/furto?

Se sim, este evento ocorreu nos últimos 12 meses?
( ) Não ( ) Sim
( ) Não ( ) Sim
( ) Não ( ) Sim

Se sim, neste evento, você sofreu violência física?

Você dirigiria depois de ter ingerido bebida alcoólica?
( ) Não tenho CNH
( ) Não
( ) Sim, já aconteceu
( ) Sim, acontece às vezes

Você emprestaria a um amigo/parente um valor equivalente à sua renda mensal (salário/mesada)?
( ) Nunca pediram
( ) Não
( ) Não, mas já emprestei
( ) Sim, mas nunca emprestei
( ) Sim, e já emprestei antes 


\section{ETAPA 1 do experimento - escala padrão}

Este experimento consiste em fazer escolhas que revelam as suas preferências sobre as opções de cada jogo. O experimento lista dez jogos (em linha) com escolhas pareadas entre "opção A" e "opção B". Você deverá escolher entre "opção A" e "opção B" para cada um dos dez jogos. Você pode escolher A para alguns jogos e B para outros, pode alternar suas escolhas ou fazê-las em qualquer ordem; o importante é que você escolha a opção de loteria que mais lhe agradaria jogar.

O experimento é hipotético, mas, se os sorteios fossem realizados, um dado de dez faces seria utilizado para determinar os seus ganhos. Este dado seria lançado 2 vezes - a primeira para sortear o jogo e a segunda para determinar o valor $(\mathrm{R} \$)$ a ser ganho para a opção que você escolheu, A ou B. Obviamente, cada jogo tem igual chance de ser sorteado (1/10) e o valor $(\mathrm{R} \$)$ a ser ganho dentro de cada opção depende da sua chance de ocorrência (1/10 ou 9/10;2/10 ou $8 / 10 ; \ldots ; 10 / 10$ ou $0 / 10)$.

Note que, se o jogo 1 for sorteado, por exemplo, a "opção A" paga R \$ 2,00 se a face sorteada do dado for 1 e paga $\mathrm{R} \$ 1,60$ se a face sorteada do dado for entre 2 e 10 . A "opção B" paga $\mathrm{R} \$ 3,85$ se a face sorteada do dado for 1 e paga $\mathrm{R} \$ 0,10$ se a face sorteada do dado for entre 2 e 10. Os demais jogos são similares, diferenciando-se pelo fato de que as chances de ganhos maiores vão aumentando. De fato, se o jogo 0 for sorteado, não é necessário lançar o dado pela segunda vez, pois este jogo paga os maiores valores com certeza, e sua escolha é entre ganhar $\mathrm{R}$ \$ 2,00 (opção $\mathrm{A}$ ) ou $\mathrm{R} \$ 3,85$ (opção B).

Então, vamos começar! Na última coluna, ESCOLHA a sua opção, A ou B.

\begin{tabular}{|c|c|}
\hline OPÇÃO A & OPÇÃO B \\
\hline $1 / 10$ de $\mathrm{R} \$ 2,00$ e $9 / 10$ de $\mathrm{R} \$ 1,60$ & $1 / 10$ de $\mathrm{R} \$ 3,85$ e $9 / 10$ de $\mathrm{R} \$ 0,10$ \\
\hline $2 / 10$ de $\mathrm{R} \$ 2,00$ e $8 / 10$ de $\mathrm{R} \$ 1,60$ & $2 / 10$ de $\mathrm{R} \$ 3,85$ e $8 / 10$ de $\mathrm{R} \$ 0,10$ \\
\hline $3 / 10$ de $\mathrm{R} \$ 2,00$ e $7 / 10$ de $\mathrm{R} \$ 1,60$ & $3 / 10$ de $\mathrm{R} \$ 3,85$ e $7 / 10$ de $\mathrm{R} \$ 0,10$ \\
\hline $4 / 10$ de $\mathrm{R} \$ 2,00$ e $6 / 10$ de $\mathrm{R} \$ 1,60$ & $4 / 10$ de $\mathrm{R} \$ 3,85$ e $6 / 10$ de $\mathrm{R} \$ 0,10$ \\
\hline $5 / 10$ de $\mathrm{R} \$ 2,00$ e $5 / 10$ de $\mathrm{R} \$ 1,60$ & $5 / 10$ de $\mathrm{R} \$ 3,85$ e $5 / 10$ de $\mathrm{R} \$ 0,10$ \\
\hline $6 / 10$ de $\mathrm{R} \$ 2,00$ e $4 / 10$ de $\mathrm{R} \$ 1,60$ & $6 / 10$ de $\mathrm{R} \$ 3,85$ e $4 / 10$ de $\mathrm{R} \$ 0,10$ \\
\hline $7 / 10$ de $\mathrm{R} \$ 2,00$ e $3 / 10$ de $\mathrm{R} \$ 1,60$ & $7 / 10$ de $\mathrm{R} \$ 3,85$ e $3 / 10$ de $\mathrm{R} \$ 0,10$ \\
\hline $8 / 10$ de $\mathrm{R} \$ 2,00$ e $2 / 10$ de $\mathrm{R} \$ 1,60$ & $8 / 10$ de $\mathrm{R} \$ 3,85$ e $2 / 10$ de $\mathrm{R} \$ 0,10$ \\
\hline $9 / 10$ de $\mathrm{R} \$ 2,00$ e $1 / 10$ de $\mathrm{R} \$ 1,60$ & $9 / 10$ de $\mathrm{R} \$ 3,85$ e $1 / 10$ de $\mathrm{R} \$ 0,10$ \\
\hline $10 / 10$ de $\mathrm{R} \$ 2,00$ e $0 / 10$ de $\mathrm{R} \$ 1,60$ & $10 / 10$ de $\mathrm{R} \$ 3,85$ e $0 / 10$ de $\mathrm{R} \$ 0,10$ \\
\hline
\end{tabular}

\begin{tabular}{c|c}
\hline JOGO & ESCOLHA \\
\hline 1 & \\
\hline 2 & \\
\hline 3 & \\
\hline 4 & \\
\hline 5 & \\
\hline 6 & \\
\hline 7 & \\
\hline 8 & \\
\hline 9 & \\
\hline 0 & \\
\hline
\end{tabular}

As escolhas são suas, então, por favor, não converse com ninguém.

Desde já, obrigado pela participação! :; 